\title{
A receptor-specific crosstalk between the prostanoid 3 and the Bombesin sub- type 3 receptors
}

\author{
Yan Zhang ${ }^{1}$, Yanfang Liu' ${ }^{2}$ Xinmiao Liang ${ }^{2}$, Olivier Civelli ${ }^{3}$ \\ ${ }^{I}$ Shanghai Jiao Tong University, China, ${ }^{2}$ Dalian Institute of Chemical Physics, China, ${ }^{3}$ University of California, Irvine, \\ USA
}

Bombesin receptor subtype-3 (BRS-3) is a G protein-coupled receptor that is expressed in the central nervous system, peripheral tissues and in tumors. Our understanding of BRS-3's role in physiology/pathophysiology is limited because its natural ligand is unknown. In an attempt to identify this ligand, we screened toad skin extracts and identified prostaglandins as putative ligands. In BRS-3-transfected HEK cells we show that prostaglandins, PGE2 being the most potent, fulfill the pharmacological criteria of affinity, selectivity and specificity to be considered as agonists to the BRS-3 receptor. However, PGE2 is unable to activate BRS-3 in different cellular environments. We speculated that endogenous prostanoid receptors (EPs) might be the cause of this cellular selectivity, and found that EP3 is the receptor primarily responsible for the differential PGE2 effect. Consequently we reconstituted the HEK environment in CHO cells and found that BRS-3 and EP3 interact to potentiate PGE2 signaling. This potentiating effect is receptor specific, and occurs only when BRS-3 is paired to EP3. Our study represents an example of functional cross-talk between two distantly related GPCRs and may be of clinical importance for BRS-3 targeted therapies. 